\title{
edmetic
}

Revista de Educación Mediática y TIC

\section{TIC y matemáticas: una integración en continuo progreso}

Una de las principales evidencias de los cambios en los sistemas educativos es la presencia de las TIC tanto en el aula como en los diseños curriculares y sU mención específica en las distintas normativas y documentos de legislación educativa.

Las TIC permiten trabajar en entornos virtuales dinámicos y de inmediatez que hacen cambiar los roles tanto del profesorado como del alumnado. Para los primeros se empieza a requerir una alfabetización o actualización tecnológica y la adaptación a sus rutinas labores al nuevo medio. Se requiere una planeación y planificación minuciosa para un uso adecuado de estas herramientas como recurso didáctico para la enseñanza y el aprendizaje.

Para los alumnos, estos pasan de un rol de espectador pasivo, a ser parte activa en el proceso de enseñanza y aprendizaje. Teniendo la posibilidad de auto regular la adquisición de conocimientos en función de sus destrezas y capacidades.

Las TIC han permitido el acceso de profesores y alumnos a recursos didácticos e información de manera casi impensable hace unos cuantos años. En el caso de las matemáticas, esta área ha sido muy beneficiada por las TIC, especialmente por el software, porque estos permiten mejorar procesos de visualización de conceptos y aseguran una adecuada compresión de ellos al ofrecer variados sistemas de representación. Esto favorece tanto el trabajo 
docente como el aprendizaje por parte del alumnado.

Por otra parte esta la posibilidad de utilizar algunas herramientas que fomentan el aprendizaje autónomo al tiempo que facilitan la atención a la diversidad del alumnado y al aprendizaje personalizado. Todo ello hace necesario que el profesorado de matemáticas conozca y domine estos recursos, así mismo debe adquirir destrezas para identificar qué le es útil a él y a sus alumnos en situaciones determinadas, bien por el tipo de contenido o por el nivel educativo en el que ejerza su labor educativa.

Para la enseñanza de las matemáticas contamos con herramientas generales, como lo son Internet, blogs libros electrónicos, WebQuests, paquetes ofimáticos, vídeos, animaciones, etc. También tenemos las herramientas específicas para las matemáticas como: calculadora, software especializado para matemáticas, applets y páginas web interactivas de matemáticas.

El constante y creciente interés por parte del profesorado de matemáticas en conocer e incorporaras en sus clases se pone en evidencia en los distintos congresos del profesorado, en los cursos de capacitación y formación permanente. Esto es importante, porque si bien toda la sociedad reconoce la utilidad de las $\mathrm{TIC}$ en los procesos educativos, es el profesorado quienes lo implementan.

Este monográfico presenta el artículo de David Arnau en el que muestra cómo la hoja de cálculo Excell puede ser utilizada por alumnos de primer curso de secundaria para resolver problemas verbales.

Nora Gatica y Oscar Ares presentan un trabajo sobre la comprensión del concepto de exactitud del método de Simpson utilizándose la interface gráfica de MATLAB, GUI para el trazo de las funciones por parte de alumnos argentinos de ingeniería.

Por su parte Miguel Villarraga et al, presentan una experiencia sobre el diseño de un curso de capacitación digital para el profesorado de matemáticas en el que se enseño a utilizar variado software matemático. Esta 
experiencia giraba en torno a los lineamientos curriculares del sistema educativo de Colombia.

El desarrollo de las competencias matemáticas señaladas por el Proyecto PISA es abordado por Ivanovnna Cruz y Ángel Puentes en un artículo en el que describen las actividades propuestas a los alumnos de la asignatura de matemática básica.

Las TIC en matemáticas de la educación primaria es el tema que presentan Antonia Ramírez y Ester Lorenzo. Su artículo despliega una serie de tareas mediadas por recursos TIC con el propósito de fomenta y desarrollar la competencia matemática.

Finalmente varios investigadores de la universidad de Córdoba presentamos un estudio sobre la participación y el fomento de competencias de cooperación entre estudiantes de primer año de Grado de Educación Infantil a través del foro virtual en la plataforma Moodle.

El monográfico recoge diversas visiones de la integración de las TIC en la enseñanza de las matemáticas y cómo el uso de herramientas tecnológicas generales y específicas convergen para facilitar y potenciar el aprendizaje de las matemáticas.

Alexander Maz-Machado

Departamento de Matemáticas

Universidad de Córdoba

edmetic, 1 (2), 2012, E-ISSN: 2254-0059; pp. 4-6 